\title{
ESTUDIO DEL COMPORTAMIENTO ALIMENTARIO INFANTIL A TRAVÉS DE LA TÉCNICA DE “DIBUJAR Y ESCRIBIR” EN ESCOLARES MEXICANOS.
}

\author{
STUDY OF CHILD FOOD BEHAVIOR THROUGH THE "DRAW AND WRITE" TECHNIQUE IN MEXICAN SCHOOLS
}

Castro Sánchez Ana Elisa*, Hernández Ruiz Karina Janett, Ramos Peña Esteban Gilberto*, Ávila Ortiz María Natividad*

*Universidad Autónoma de Nuevo León (UANL), México.

Citation: Castro Sánchez AE, Hernández Ruiz KJ, Ramos Peña EG, Ávila Ortiz MN. (2017) Estudio del comportamiento alimentario infantil a través de la técnica de "dibujar y escribir" en escolares mexicanos. Revista de Salud Pública y Nutrición, 16(3), 6-13.

Editor: Esteban G. Ramos Peña, Dr. CS., Universidad Autónoma de Nuevo león, Facultad de Salud Pública, Monterrey Nuevo León, México.

Copyright: (2017 Castro Sánchez AE et al. This is an open-access article distributed under the terms of Creative Commons Attribution License [CC BY-ND 4.0], which permits unrestricted use, distribution, and reproduction in any medium, provided the original author and source are credited.

Competing interests: The authors have declared that no competing interests exist.

DOI: https://doi.org/10.29105/respyn16.3-2

Email: ana.castrosh@uanl.edu.mx, ana.castros@uanl.mx 


\title{
ESTUDIO DEL COMPORTAMIENTO ALIMENTARIO INFANTIL A TRAVÉS DE LA TÉCNICA DE "DIBUJAR Y ESCRIBIR" EN ESCOLARES MEXICANOS
}

\author{
Castro Sánchez Ana Elisa*, Hernández Ruiz Karina Janett*, Ramos Peña Esteban Gilberto*, Ávila Ortiz María \\ Natividad*
}

\author{
*Universidad Autónoma de Nuevo León (UANL), México
}

\section{RESUMEN}

Introducción: México presenta una de las prevalencias más altas en obesidad infantil a nivel mundial. Sin embargo poco se sabe sobre el estilo de vida relacionado con la alimentación de los niños y niñas mexicanos. Objetivo: Estudiar el comportamiento alimentario en niños y niñas mexicanos a través de la técnica cualitativa de "dibujar y escribir" Métodos: investigación cuantitativa-cualitativa; participaron 186 estudiantes. Las respuestas se registraron como texto; se agruparon en categorías y se cuantificaron; se elaboraron tablas de frecuencia; se realizaron pruebas estadísticas. Resultados: se obtuvo un total de 2,727 dibujos con su descripción; todos los grados presentaron diferencias significativas $(p<0.001)$. Conclusiones: La prueba manifiesta mayor eficiencia en los grados escolares de cuarto y quinto de primaria; en secundaria ya no es eficiente. Los resultados indican pistas útiles para ser utilizadas en las estrategias de orientación alimentaria y en los programas de atención y control de la obesidad infantil.

Palabras Clave: técnica "dibujar y escribir"; escolares mexicanos; comportamiento alimentario.

\section{ABSTRACT}

Introduction: Mexicans are among the first in the world to address a high prevalence of overweight and obesity coupled with malnutrition in their population. However, little is known about the lifestyle of Mexican children with respect to food. Objective: The study objective was to investigate the eating behaviour of Mexican children using the qualitative technique of "Drawing and Writing." Methods: mixed (quantitative-qualitative) study. The trial was conducted with 186 students from pre-school to the first year of secondary school. Responses were recorded as text; they were grouped into categories and quantified; frequency tables were developed; statistical analyzes were performed. Results A total of 2,727 drawings with descriptions were collected, and the data showed significant differences $(p<0.001)$ in general. Conclusions: The technique used in the trial had the greatest effectiveness in the fourth and fifth grades of primary school, whilst in secondary school, it was no longer effective.

Key words: Mexican students; "Drawing and Writing" technique; eating behaviour 


\section{Introducción}

En el último cuarto de siglo han surgido importantes cambios derivados de la globalización; de entre los cuales destacan, las transformaciones demográficas, socioculturales, tecnológicas y económicas a nivel mundial. Dichos cambios han generado un impacto considerable en México con respecto a la transformación del perfil epidemiológico, los estilos de vida, los patrones de consumo alimentario y actividad física (Gómez, et. al. 2016), éstos dos últimos, generando un aumento global de malnutrición principalmente por exceso.

De acuerdo con la Obesity Update (2017) más de la mitad de los adultos y casi 1 de cada 6 niños padecen sobrepeso u obesidad en países asociados a la Organización para la Cooperación y el Desarrollo Económico (OCDE), siendo México una de las naciones con mayor obesidad en adultos en el mundo.

Según datos de la Encuesta Nacional de Salud y Nutrición (ENSANUT) 2016, el $71.2 \%$ de la población adulta presenta sobrepeso u obesidad, mientras que dicha problemática afecta al 33.2\% de los niños en edad escolar (5-11 años).

Por lo anterior, se ha puesto atención especial en el estudio de los hábitos alimentarios de las poblaciones debido al aumento de las problemáticas derivadas de la malnutrición por exceso como lo son, la obesidad (OB) infantil y las enfermedades crónico degenerativas a temprana edad, lo que se ha convertido en un grave problema de salud pública por los altos costos que se derivan de su atención y por las repercusiones en la vida adulta.

Podemos darnos cuenta que si bien, México cuenta con datos de salud y nutrición derivados de encuestas nacionales $y$, particularmente Nuevo León, que cuenta con la Encuesta Estatal de Alimentación y Nutrición, de acuerdo con Ramos (2015) dicha información posee desventajas metodológicas; por ejemplo, el hecho de que la información recabada corresponde a la familia en general, no considera la distribución de alimentos por edad y género al interior del hogar y se basa en percepciones o creencias acerca de los hábitos y costumbres alimentarias de los miembros. Además, cabe destacar que los niños en edad escolar suelen pasar una parte importante del día lejos de la vista vigilante de sus padres, ya sea en la escuela o en actividades recreativas y posiblemente consuman alimentos fuera del conocimiento de ellos.

Por ello diversos estudios ya se encuentran enfocados en abordar las causas inmediatas que originan el SP y OB infantil, como lo son el desequilibrio de energía, asociado con dietas hipercalóricas; la calidad de macro y micronutrientes; la actividad física, como se mencionó previamente; y otros factores como el acceso y las fuentes de los alimentos, las políticas de mercadeo y publicidad y los ambientes generadores de obesidad (Shamah, Amaya, \& Cuevas, 2015).

Si bien, podemos indagar en estudios enfocados al conocimiento y causas que originan la adquisición de hábitos alimentarios positivos o negativos en niños, sin embargo, uno de los aspectos más complejos es el de la obtención de datos directamente de los niños y niñas sin la intermediación del jefe de familia. Lo anterior debido a que históricamente niñas y niños han sido vistos como objetos de estudio al considerarlos incompetentes o poco confiables, con limitada capacidad verbal, e incapaces de recuperar información o entender preguntas. Sin embargo, ellas y ellos tienen su propia perspectiva sobre los aspectos que influyen en su vida y entorno social y esta información se obtiene sobre todo a través de un contacto directo y empleando métodos y técnicas cualitativas.

No se han identificado trabajos de investigación nutriológica que hayan utilizado la técnica cualitativa de "dibujar y escribir" en población mexicana, esto a pesar de que el empleo de dibujos o mapas es un método aplicado desde hace varias décadas en diversos grupos poblacionales (Mauthner, Mayall, \& Turner, 1993; Box \& Landman, 1994) destacando los estudios de Charlton (1996), Bendelow \& Oakley (1994) y el de Pridmore \& Bendelow (1994) en África.

Recientemente ha tomado más fuerza en la investigación con niños, para obtener datos de las percepciones y espacios en donde éstos se desenvuelven, y de esta manera obtener una mejor comprensión de la vida infantil particularmente de lo que ellos relacionan como saludable o no saludable (O’Connell, 2013; Bridal, Hendrie, Thompson \& Blunden, 2012). 
Dicha técnica consiste en un método de investigación visual basado en el arte de dibujar y escribir, en donde se solicita a los participantes que realicen una actividad de dibujo junto con un ejercicio de escritura, que complementa la comprensión del dibujo. Comúnmente dicha técnica es administrada a los niños por profesores o investigadores en el aula (Hartel, 2014).

Dentro de las ventajas que tiene el empleo de esta técnica se encuentran, por un lado, que los niños en edad escolar están familiarizados con las herramientas y materiales necesarios para la elaboración de dibujos y la descripción de los mismos; por lo tanto es un método válido para obtener datos directamente de ellos mismos (Horstman, Aldiss, Richardson, \& Gibson, 2008).Por otro lado, "dibujar y escribir" es una técnica cualitativa que también permite hacer análisis cuantitativos o mixtos por ejemplo, complementando el análisis de texto y descriptivo con diversas pruebas estadísticas (Piko \& Bak, 2006).

Se obtiene información más enriquecedora debido a que cuando a los niños se les solicita dibujar y contar sobre algo en específico, revelan el doble de información, esto a diferencia de que si se les solicitara verbalmente (Walker, Caine-Bish \& Wait, 2009). Lo anterior concuerda con el estudio de Horstman et al (2008) quienes reportaron que, al ser considerado una actividad divertida, los niños proporcionan datos más enriquecedores que si sólo se les pidiera escribir o hablar sobre algún tema, además de que disfrutan la realización de dibujos.

Ahora bien, una de las desventajas u observaciones a esta técnica, se centran en los protocolos éticos relacionados con el consentimiento, privacidad y el uso de las imágenes después del estudio, por otra parte se menciona que el ejercicio de dibujo resulta desagradable para una minoría de sujetos (Hartel, 2014).

A pesar de las desventajas que esta técnica pueda tener, estudios demuestran que son más los datos significativos que se obtienen al ser una técnica atractiva para los niños $\mathrm{y}$, lo que particularmente nos interesa es el empleo de dicha técnica en población mexicana y conocer la percepción de los niños en cuanto a gustos y hábitos alimentarios y conocimiento de alimentos saludables y no saludables. El objetivo general ha sido estudiar el comportamiento alimentario en niños y niñas mexicanos a través de la técnica cualitativa de “dibujar y escribir”. Específicamente, se determinó cuál es el grupo de edad de máxima eficiencia para responder la técnica; el porcentaje de no respuesta; aceptación de la prueba; cantidad de información generada por temas, grupos de edad y estado nutricio; y dificultades durante la aplicación de la prueba.

\section{Material y Métodos}

Entre el año 2011 y 2013 se realizó un estudio mixto (cualitativo-cuantitativo) transversal. Previamente se obtuvo la aprobación del Comité de Ética e Investigación de la Facultad de Salud Pública y Nutrición de la Universidad Autónoma de Nuevo León (Folio de aprobación: 11-FaSPyN-SO-03), el permiso de las autoridades educativas, el consentimiento informado de los padres y madres de familia y el asentimiento de los escolares.

Las consideraciones éticas se realizaron con apego a las disposiciones dictadas en el Reglamento de la Ley General de Salud en Materia de Investigación (Secretaría de Salud [SS], 1987), específicamente en los siguientes apartados: el Capítulo I, Artículo 13 menciona que en la investigación en donde el ser humano sea el sujeto de estudio, debe prevalecer el respeto a la dignidad y protección de los derechos y bienestar.

En el presente estudio se respetó el derecho de decisión de participar tanto de los padres de familia como de los propios niños. El Artículo 14, fracción IV; hace mención que se deberá mantener la relación riesgo beneficio. En este proyecto, si bien los beneficios se limitan a la detección de malnutrición (exceso o deficiencias) y la remisión con los especialistas de acuerdo a la afiliación de padres, no existen riesgos derivados de las técnicas de investigación: ya que sólo se procedió a la toma de mediciones antropométricas de los niños así como ilustrar a través del dibujo una serie de imágenes sobre hábitos y preferencias alimentarias.

El estudio se realizó previo a que la escuela adoptara las medidas gubernamentales para el control de alimentos y bebidas en las instituciones de educación básica. 
Participaron 102 niñas y 84 niños cursando entre tercero de preescolar y primero de secundaria, en una escuela privada ubicada en un sector urbano de nivel socioeconómico medio.

En una primera etapa se tomaron datos antropométricos (peso, talla, IMC) por personal calificado de nutrición. Para determinar el Índice de Masa Corporal (IMC), para la edad se obtuvo el punto de corte puntaje $\mathrm{z}$ y se procedió a la interpretación de diagnósticos, que de acuerdo a la Organización Mundial de la Salud (2007): el estado nutricio Adecuado, tiene un puntaje de $0,<-1.0$, sobrepeso $>1.0$, Obesidad $>2.0$ Obesidad severa $>3.0,<-2.0$ Delgadez y $<-3.0$ Delgadez severa.

En la segunda etapa se aplicó la técnica de "dibujar y escribir". En cada grupo de escolares se contó con el apoyo de personal entrenado compuesto por: 2 psicólogas, 1 profesora, 1 pedagoga y 2 nutriólogas. Los niños y niñas se encontraban en sus respectivos salones de clase sentados en escritorios individuales y se les proporcionaron hojas blancas y lápices de colores para realizar sus dibujos.

El instrumento para dibujar y escribir consistía en 4 hojas bond tamaño oficio engrapadas, en la primera hoja se les solicitó dibujar los lugares donde acostumbraba salir a comer con su familia; en la segunda, sus comidas y bebidas favoritas, lo que come en la escuela, con quién le gusta comer; en la tercer hoja, cuáles alimentos consideraba saludables $\mathrm{y}$, en la cuarta hoja los alimentos que consideraba no saludables. Se les explicó que no se calificaría la habilidad de dibujar y que eran libres de plasmar cualquier imagen que consideraran como respuesta correcta (Walker, Caine-Bish \& Wait, 2009).

Una vez que completaron los dibujos, una entrevistadora del equipo de apoyo tomaba notas al momento de solicitar al niño o niña que describiera su dibujo: "Cuéntame qué dibujaste". Luego, seguían otras preguntas, como: ¿Por qué dibujaste esto? ¿Qué quieres decir con esto? ¿Hay algo más que quieras contarme acerca de tu dibujo? También se les pidió que escribieran un nombre o título a cada uno de sus dibujos (Walker, Caine-Bish \& Wait, 2009). En el caso de los escolares de tercer grado de preescolar y primero de primaria, se les apoyó con la escritura. Las entrevistas tuvieron una duración de entre 10 y 30 minutos.
Las respuestas se registraron como texto; se agruparon en categorías y se cuantificaron; se elaboraron tablas de frecuencia. También se realizaron pruebas de Chi cuadrada.

\section{Resultados}

Se obtuvo un total de 2,727 dibujos con su descripción. La prueba se aplicó a 186 estudiantes con escolaridad desde preescolar hasta primero de secundaria; en general todo los grados presentaron diferencias significativas $(\mathrm{p}<0.001)$. De ellos, el $16.15 \%$ estaba en preescolar, $78.5 \%$ en primaria y el $5.9 \%$ en secundaria. En promedio, la menor cantidad de respuestas fue aportada por el grado de secundaria $(3.6 \pm 1.6)$, mientras que los estudiantes del cuarto $(17.1 \pm 3.5)$ y de quinto $(17.5 \pm 3.4)$ año de primaria fueron los que más información aportaron (Tabla 1).

\begin{tabular}{|c|c|c|c|}
\hline Gado & $\mathrm{n}$ & Media & DS \\
\hline $\begin{array}{l}\text { Preescolar } \\
\text { Primaria }\end{array}$ & 29 & 16.1 & 3.5 \\
\hline 1 & 29 & 14.2 & 1.6 \\
\hline 2 & 33 & 13.6 & 2.1 \\
\hline 3 & 32 & 14.3 & 1.9 \\
\hline 4 & 16 & 17.1 & 3.5 \\
\hline 5 & 24 & 17.5 & 3.4 \\
\hline 6 & 12 & 16.3 & 2.1 \\
\hline \multicolumn{4}{|l|}{ Secundaria } \\
\hline 1 & 11 & 3.6 & 1.6 \\
\hline
\end{tabular}

En cuanto a los resultados del número de respuestas en razón del género de los estudiantes, en general no hay diferencias significativas entre los diferentes estados de nutrición $(\mathrm{p}<0.05)$. El mayor porcentaje de estudiantes $(58.1 \%)$ se encontraban en estado nutricio adecuado y el $38.2 \%$ en sobrepeso y obesidad (Tabla 2). 
técnica "dibujar y escribir"; escolares mexicanos; comportamiento alimentario

Tabla 2.- Frecuencias, medias y desviación estándar de resupuestas según estado nutricio

\begin{tabular}{lrcc}
\hline Edo. Nutricio & $\mathrm{n}$ & Media & DS \\
\hline Delgadez & 1 & 15.0 & - \\
Adecuado & 108 & 15.0 & 3.1 \\
Riesgo de sobrepeso & 6 & 17.2 & 2.4 \\
Sobrepeso & 36 & 13.9 & 4.6 \\
Obesidad & 24 & 14.1 & 2.1 \\
Obesidad severa & 11 & 16.1 & 2.9 \\
\hline
\end{tabular}

p $>0.05$

Fuente: Entrevista

También, entre lo más destacable de los resultados se encuentra que los niños identifican con facilidad como alimentos saludables a las frutas y verduras (65\% de los dibujos) y como alimentos no saludables a las frituras, dulces/postres (43\% de los dibujos) (Figura 1).

\section{Figura 1.- Alimentos saludables y no saludables}
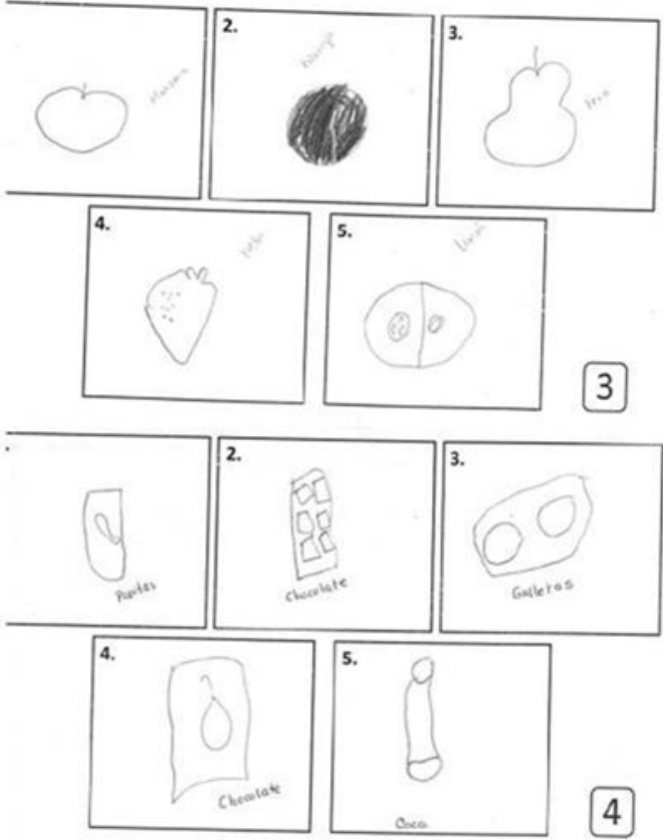

Entre los lugares que frecuentan cuando no comen en casa, destacan las hamburgueserías y los restaurantes con servicio completo. En cuanto a la comida y bebida dentro del plantel escolar, destaca el consumo de sándwiches/tortas y el jugo industrializado. Sobre los lugares preferidos o dónde les gusta más ir a comer, son las hamburgueserías y pizzerías (Figura
2). Mientras que las pastas/harinas y las bebidas gaseosas azucaradas aparecen también como las preferidas y esto independientemente del estado nutricio y del género. Por otro lado, aparece que comer en compañía de los miembros de la familia, es la preferencia más señalada (Figura 2).

\section{Figura 2.- Hábitos y preferencias alimentarias}

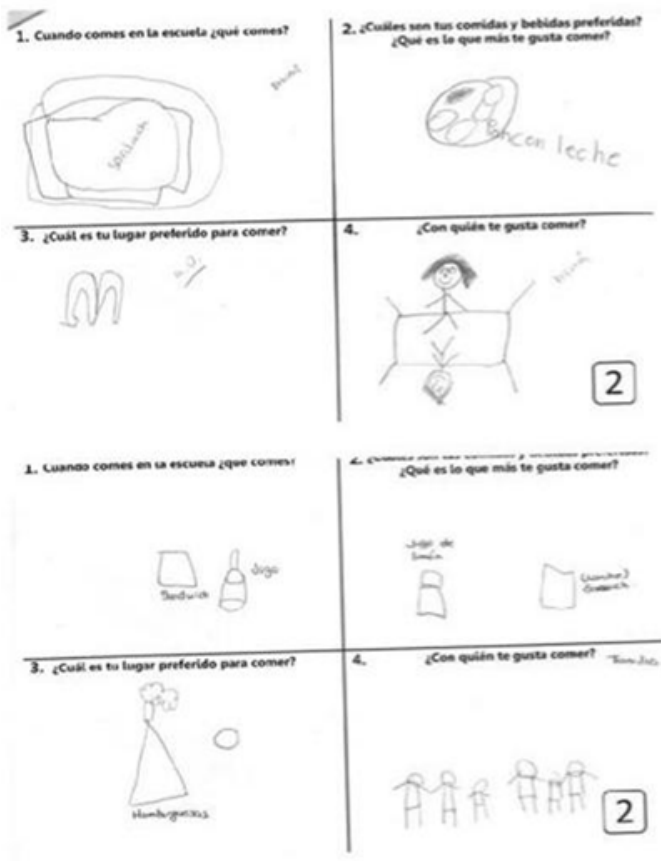

En cuanto al tiempo que se tardaron en completar el instrumento, los participantes de entre tercero de preescolar y segundo de primaria (5-6 años) tardaron entre 40 y 50 minutos en completar el instrumento; mientras que, los de $3^{\circ}$ de primaria y hasta $1^{\circ}$ de secundaria (7-12 años) entre 20 y 30 minutos. En preescolar, niños y niñas manifestaron cansancio en la mano por dibujar y escribir largo rato.

Desde el análisis cualitativo un hallazgo importante se refiere a que en preescolar y $1^{\circ}$ de primaria algunos aún no distinguen entre productos comestibles y no comestibles. En cuanto al género, sólo niñas reportaron consumir ensaladas y por el contrario sólo varones reportaron mariscos. Además en $1^{\circ}$ de primaria solicitaban más tiempo para colorear sus dibujos. En $1^{\circ}$ de secundaria, hubo negativa a 
comunicar las ideas mediante el dibujo, manifestando preferencia por la escritura y la entrevista.

\section{Discusión}

"Dibujar y Escribir" es una técnica cualitativa que permite hacer análisis mixtos, por ejemplo, complementando el análisis de texto y descriptivo con pruebas estadísticas. Es un método aplicado desde hace varias décadas en diversos grupos poblacionales destacando Inglaterra, África y Canadá. "Dibujar y escribir" permite obtener una mejor comprensión de la vida infantil.

El presente estudio mixto extiende la literatura existente sobre el uso de la técnica de "Dibujar y Escribir" al aportar datos sobre el estudio del comportamiento alimentario en población mexicana. Aporta resultados cualitativos y cuantitativos sobre el estudio del comportamiento alimentario de una población infantil que presenta de las más altas prevalencias de sobrepeso y obesidad en el mundo. Los resultados permiten reducir el vacío de conocimiento sobre esta población específica (Ramos et al, 2015), además de ampliar la literatura existente sobre el uso de distintos instrumentos de medición y de colecta de datos en menores de edad sin la intermediación de un adulto.

Ahora bien, el contraste de resultados sólo puede realizarse en cuanto a la utilización de la técnica y no propiamente de los datos obtenidos debido a que no existen estudios realizados específicamente en el área de alimentación y nutrición, por lo que existe un vacío de conocimiento que debe ser abordado por especialistas de la nutrición en México.

Los estudios presentados a continuación muestran resultados semejantes sólo en un apartado de la totalidad de su estudio debido a que no se enfocaron en sí en percepción de alimentos saludables y no saludables si no en el concepto de salud y enfermedad en general.

En el estudio de Cetin, Ozarslan, Isik y Eser (2012) realizado en Turquía en estudiantes de $9^{\circ}$ grado (14 años) se les solicitó dibujar y escribir sobre nutrición y refrigerios y se obtuvieron 22 explicaciones escritas y cero dibujos, lo que coincide parcialmente con nuestro estudio pues los estudiantes de secundaria (13 - 15 años) sólo aportaron el 5.9\% de dibujos y mencionaron verbalmente su negativa a expresar a través del dibujo, sus respuestas. Asimismo de los 156 estudiantes del estudio Turco, sólo obtuvieron 289 dibujos en total, lo que representa $1.8 \%$ de dibujos en promedio. Cabe destacar que los otros aspectos estudiados fueron definición del concepto de salud, factores protectores de la salud, factores que afectan negativamente la salud, cuerpo humano y medicina.

Mientras que los resultados del estudio de Bridal, Hendrie, Thompson y Blunden (2012) efectuado en Australia, en donde obtuvieron la percepción de 28 niños y niñas de entre 5 a 9 años de lo que es saludable y no saludable; obtuvieron 11 dibujos relacionados a alimentos saludables siendo sólo frutas lo que plasmaron. Por lo tanto, al igual que en nuestro estudio los niños y niñas relacionan más fácilmente como alimentos saludables, las frutas y verduras (65\% de los dibujos).

En un estudio efectuado en Portugal por Lima y Serra (2014) en donde emplearon la técnica de dibujar y escribir en 209 niños de 10 a 12 años para indagar sobre conceptos de salud y enfermedad encontraron que en la categoría de definición de saludable, los niños plasmaron dibujos relacionados a consumo de alimentos adecuados como frutas y bajo consumo de dulces, lo que se asemeja a los resultados de nuestro estudio en cuanto a la relación de alimentos saludables.

En cuanto a la aceptabilidad de la técnica de dibujar y escribir, de los 186 niños y niñas que participaron en nuestro estudio, el $6 \%$ de los datos obtenidos correspondieron a niños de entre 13 a 14 años, mientras que en el estudio de Noonan, Boddy, Fairclough y Knowles (2016) de los 181 niños y niñas de entre 10 a 11 años, sólo 30 de ellos (16\%) completaron la tarea de dibujar y escribir con relación a la práctica de actividad física.

Knighting, Rowa-Dewar, Malcolm, Kearney y Gibson (2011) llevaron a cabo un estudio empleando la técnica, indagando sobre la comprensión de los niños sobre el cáncer, incluyendo 195 niños de entre 10 a 11 años, destacando en una de las categorías comportamientos saludables y no saludables, resultando 253 dibujos de alimentos saludables y 80 dibujos de bebidas saludables, destacando que en 
nuestro estudio el $65 \%$ de los dibujos fueron para alimentos saludables.

Podemos darnos cuenta que son pocos los resultados que podemos contrastar con otros estudios, esto debido al vacío de conocimiento que existe en México, específicamente en lo relacionado a estudios de la percepción, gustos y preferencias alimentarias en niños.

De manera general, los resultados obtenidos en nuestro estudio destacan, en cuanto al género de los participantes, se identificaron diferencias sólo de tipo cualitativo, pero que no fueron estadísticamente significativas, coincidiendo esto último con los resultados de Piko \& Bak (2006).

Los resultados obtenidos por parte de los niños fueron muy enriquecedores y brindaron al equipo de investigadores un amplio panorama de los hábitos que tienen los niños así como sus gustos y preferencias alimentarias.

La mayor cantidad de dificultades para responder el instrumento se presentaron entre 3ro de preescolar y 20 de primaria, además de que éstos se tardaron el doble de tiempo que el resto de los participantes. Se obtuvo una mayor cantidad de imágenes en $4^{\circ}$ y $5^{\circ}$. A partir de primero de secundaria prefieren el cuestionario y la entrevista. Los resultados demuestran que la prueba manifiesta mayor eficiencia en los grados escolares de cuarto y quinto de primaria, mientras que, en secundaria ya no es eficiente.

\section{Conclusiones}

Los resultados de este estudio abren la posibilidad de una utilización más eficaz de la técnica cualitativa en cuestión; y se sugieren pistas para hacer adaptaciones pertinentes según la edad y grado de la población escolar. Por ejemplo, los resultados apuntan a que la distinción entre lo comestible y no comestible queda más claro a partir del primer grado de primaria, es decir, después de los 6 años de edad, y con respecto al objetivo de la investigación, el grupo de edad de máxima eficiencia para responder la técnica fueron los de cuarto y quinto grado $(9-10$ años de edad) debido a que fueron los que más información aportaron.

La técnica de "dibujar y escribir" ha sido empleada frecuentemente en el área de la salud, sin embargo, muy poco se ha aplicado en el área de la nutrición y alimentación. Esto a pesar de que como se ha mostrado, esta técnica cualitativa permite obtener datos muy enriquecedores y significativos, al aportar conocimiento sobre el punto de vista y percepción de los niños. Lo anterior, no debería ser minimizado en su utilización en la planeación de programas de intervención, diseño de menús, entre otras cosas. Contar con la participación e interacción de los niños podría redundar en un mayor y mejor impacto en orientación alimentaria y en educación para la salud. Finalmente, sería deseable que estas técnicas cualitativas se aplicaran dentro de una estrategia multi-método.

\section{Bibliografía}

Box, V., \& Landman, J. (1994). Children Who Have No Breakfast. Health Education, 10-13.

Brindal, E., Hendrie, G., Thompson, K., \& Blunden, S. (2012). How do Australian junior primary school children perceive the concepts of "healthy" and "unhealthy"? Health Education, 406-420.

Cetin, G., Ozarslan, M., Isik, E., \& Eser, H. (2012). Students' views about health concept by drawing and writing technique. Energy Education Science and Technology, 311-316.

Charlton, A. (1996). Children and smoking: the family circle. British Medical Bulletin, 90-107.

Gómez, G., Gutiérrez, P., Viveros, J., Pérez, M., Miranda, M., Soria, C., \& Gutiérrez, O. (2016). Prevalencia de sobrepeso u obesidad en escolares de una población urbana del estado de Jalisco, México. European Scientific Journal, 62-73.

Hartel, J. (2014). An Arts-Informed Study of Information Using the Draw-and-Write Technique. JOURNAL OF THE ASSOCIATION FOR INFORMATION SCIENCE AND TECHNOLOGY, 1349-1367.

Horstman, M., Aldiss, S., Richardson, A., \& Gibson, F. (2008). Methodological Issues When Using the Draw and Write Technique With Children Aged 6 to 12 Years. Qualitative Health Research, 1001-1011.

Knighting, K. R.-D. (2011). Children's understanding of cancer and views on health-related behaviour: a 'draw and write' study. Child: Care, health and development, 289-299. 
Lima, L., \& Serra, M. (2014). The Importance of the Instructions in the Use of Draw-and-Write Techniques for Understanding Children's Health and Illness Concepts. Psychology, Community \& Health, 146- 157.

Mauthner M., M. B. (1993). Children and Food at Primary School. Social Science Research Unit.

Noonan, R., Boddy, L., Fairclough, S., \& Knowles, Z. (2016). Write, draw, show, and tell: a child-centred dual methodology to explore perceptions of out-ofschool physical activity. BMC Public Health, 1-19.

Oakley, A., Bendelow, G., Barnes, J., \& Buchanan, M. (1995). Health and cancer prevention: knowledge and beliefs of children and young people. The BMJ.

OECD. (2017). Obesity Update. Obtenido de http://www.oecd.org/els/health-systems/ObesityUpdate-2017.pdf

OMS. (2007). Organización Mundial de la Salud. Obtenido http://www.who.int/childgrowth/standards/imc_para edad/es/

OMS. (Septiembre de 2015). Organización Mundial de la Salud. Obtenido de http://www.who.int/mediacentre/factsheets/fs394/es/

Piko, B. B. (2006). Children's perceptions of health and illness: images and lay concepts in preadolescence. Health Education Research, 643-653.

Pridmore, P., \& Bendelow, G. (1995). Images of health: exploring beliefs of children using the "draw-andwrite" technique. Health Education Journal, 473-488.

Pública, I. N. (2016). Encuesta Nacional de Salud y Nutrición de Medio Camino. Obtenido de http://promocion.salud.gob.mx/dgps/descargas 1/docto s_2016/ensanut_mc_2016-310oct.pdf

Ramos, E., Curiel, E., Castro, A., Salinas, A., Núñez, G., Ramírez, E., \& De la Garza, Y. (2015). Discrepancia de la información obtenida por un mismo cuestionario de frecuencia alimentaria por dos métodos. Cultura, Tecnología y Patrimonio, 91-108.

Salud, L. G. (1987). Reglamento de la Ley General de Salud en Materia de Investigación para la Salud. Obtenido de http://www.salud.gob.mx/unidades/cdi/nom/compi/rlg smis.html
Shamah, T., Amaya, M., \& Cuevas, L. (2015) Desnutrición y obesidad: Doble carga en México. Revista Digital Universitaria UNAM, 1-14.

Walker, K., Caine-Bish, N., \& Wait, S. (2009). I like to jump on my trampoline: An analysis of drawings from 8 to 12 year old children. Beginning a WeightManagement Program. Qualitative Health Research, 906-917. 